\title{
Mucin Embolism to Cerebral Arteries: A Fatal Complication of Carcinoma of the Breast
}

\author{
JOHN H. N. DECK AND MARY A. LEE
}

SUMMARY: The case is reported of a woman with a mucin producing lobular carcinoma of the breast with metastases to many bone sites, whose terminal neurological illness was the result, not of cerebral metastases, but of cerebral infarcts. These were caused by emboli of mucin and emulsified fat, originating in bone metastases.

The pathogenesis of this embolism is compared with that of traumatic fat embolism. Attention is drawn to this process because emboli of this type have never been reported and because this distant nonmetastatic effect of carcinoma may have been overlooked in other cases. It is suggested that this mechanism should be considered in the diagnosis of otherwise $o b$ scure cerebral infarction.

RESUMÉ: Nous rapportons le cas d'une femme qui était porteuse d'un carcinome lobulaire du sein producteur de mucine. Cette tumeur essaimait de nombreuses métastases aux os, mais la maladie neurologique terminale ne fut pas le résultat de métastases cérébrales, mais plutôt d'infarctus cérébraux. Ceux-ci furent dûs à des embolies de mucine et de gras émulsifiés originant des métastases osseuses.

La pathogénèse de ces embolies est comparée $\dot{a}$ celle des embolies graisseuses traumatiques. Nous attirons l'attention sur cette forme d'embolie qui n'a jamais été rapportée et parce que cet effet nonmétastatique à distance a pû être négligé auparavant. Nous suggérons que ce type de mécanisme devrait être considéré plus souvent lors d'infarctus cérébraux obscurs.

From: The Department of Pathology, The Toronto Western Hospital.

Presented at the Annual Meeting of the Canadian Association of Neuropathologists, October 1, 1977 at Vancouver, B.C.

Reprint requests to: John H. N. Deck, M.D., Department of Pathology, Toronto Western Hospital, 399 Bathurst Street, Toronto, Canada M5T 2 S8.

\section{INTRODUCTION:}

Neurological complications of malignant disease are usually the direct result of metastasis to the central or peripheral nervous system. Less commonly they result from non-metastatic mechanisms only some of which are understood. (Brain and Adams, 1965, Wilkinson, Croft and Urich, 1967). The case here reported is one of lobular carcinoma of the breast in which, despite the presence of extensive generalized metastases, the terminal neurological deterioration was caused not by metastases to the brain, but by cerebral infarcts. Emboli of a type not previously reported were the cause of these infarcts. The emboli were composed of mucin and a small amount of emulsified fat and were devoid of tumor cells.

The case report documents a type of embolus not previously recognized and can be viewed as a previously unrecognized non-metastatic distant effect of malignancy.

\section{CLINICAL SUMMARY:}

A 63 year old right-handed woman underwent right radical mastectomy for lobular carcinoma of the breast in April 1970. Axillary lymph node metastases were present at that time. She was discharged from hospital on fluoxymesterone $10 \mathrm{mg}$ b.i.d.

She remained well until the onset of back pain in October, 1972 when she was found to have radiographic evidence of osteoblastic metastases to the thoracic vertebrae. By March, 1973 she had further evidence of bony metastases and developed tumor nodules in the skin of the abdominal wall. Her therapy was changed to 5fluorouracil at 3 to 4 week intervals, resulting in marked reduction of bone pain and disappearance of the skin nodules. Glucocorticoid $20 \mathrm{mg}$ daily was added to her regimen in June, 1974. By December, 1975 she had symptomatic and radiographic evidence of extensive bony metastases involving cervical, thoracic, and lumbar vertebrae, the skull, ribs, scapulae, and pelvis. Therapy was again changed to cyclophosphamide, vincristine sulphate, doxorubicin (Adriamycin) and an increased dose of glucocorticoid.

In August, 1976, the gradual onset of dysphasia prompted her final admission to hospital where she was found to be alert but with mild expressive dysphasia and with right hemianopia or hemi-inattention. She also had a mild left hemiparesis involving face, arm and leg with left hyperreflexia and a left Babinski response. She soon became extremely restless, agitated, and confused and within 48 hours became completely mute with total receptive and expressive aphasia. The left hemiplegia worsened and dysphagia appeared.

Routine hematological investigations were normal. Platelet count was 170,000 per cu mm. A brain scan and left carotid angiogram were reported normal. E.E.G., however, showed diffuse bilateral abnormalities with distinctive areas of slower activity in the left parieto-temporal and the right parietal regions. Treatment with dexamethasone was ineffective and she continued to deteriorate and died eleven days after the onset of her neurological symptons.

\section{POST MORTEM FINDINGS:}

Gross findings: Metastatic carcinoma was present as numerous small nodules in the liver and spleen, on the serosal surface of the stomach, in mediastinal and retroperitoneal lymph nodes, in both ovaries and as widespread bony deposits in spine, sternum, ribs and skull, which were the only bony sites examined. The bony metastases were grossly characterized by mottled discoloration of bone marrow areas, some patches showing an osteosclerotic pattern while others were 


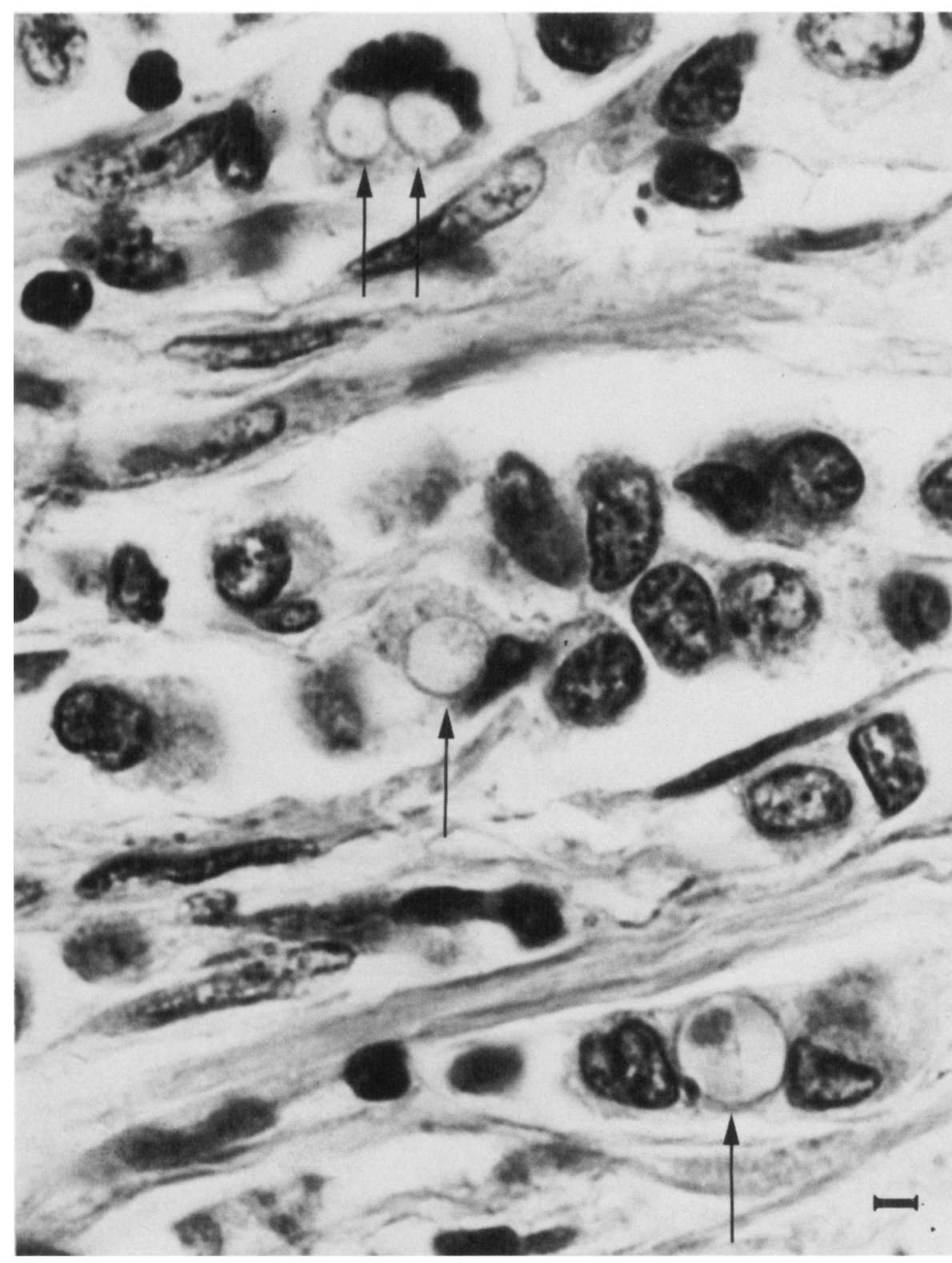

Figure 1-Primary infiltrating lobular carcinoma of breast. Note cytoplasmic vacuoles (arrows) filled with pale basophilic material. Histologically identical tumor was present in the metastases. H\&E $\times 1000$

greyish, glary and translucent. The spleen was of normal size (170 grams) and in addition to the tumor nodules was of firm consistency and tough to cut. No tumor deposits were identified in the lungs, heart, kidneys or adrenal glands on gross examination. The adrenals exhibited cortical atrophy in keeping with long term corticosteroid therapy. The heart was free of localized lesions of the myocardium, the valves were normal in appearance and free of vegetations, and no mural thrombi were present in any chamber.

The brain showed slight patchy recent subarachnoid hemorrhage over the left parietal convexity and external features of slight enlargement with bilateral flattening of cerebral gyri, but without shift of midline structures and with no transtentorial or foramen magnum herniation. Coronal sections of the brain revealed numerous recent hemorrhages up to $4 \mathrm{~cm}$ in diameter of cerebral cortex and white matter, basal ganglia, thalamus and cerebellum (Fig. 2). Some of these hemorrhages were clearly present in relation to areas of recent infarction but no tumor deposits could be identified on gross examination.

Microscopic findings: The tumor

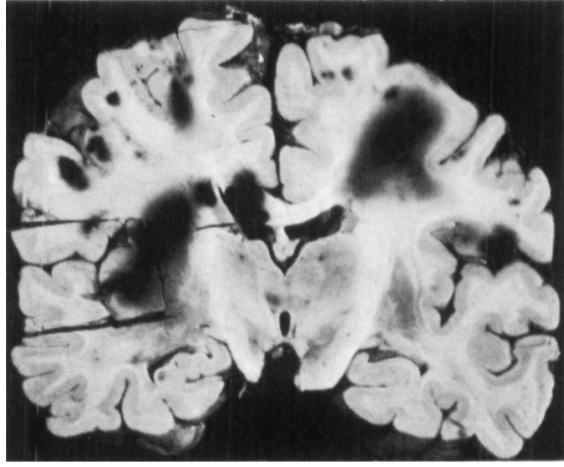

Figure 2-Coronal section of brain at autopsy. Note multiple ill-defined hemorrhagic lesions of variable size. Similar lesions were present in all areas of the cerebrum and also in the brain stem and cerebellum.

deposits at autopsy were strikingly similar to those of the original mastectomy specimen which is shown in Fig 1. The tumor cells were small with eccentric nuclei and with cytoplasmic vacuoles filled with mucicarminepositive, PAS-positive, alcian-bluepositive material. The original diagnosis of invasive mucin-secreting lobular carcinoma of the breast was considered to be supported by these findings.

Extensive histological examination of the brain showed numerous recent infarcts of 1 to 2 weeks age, many of which were hemorrhagic. Many of the nearby small cerebral arteries, arterioles, and capillaries (Fig $3 \mathrm{~A}$ and Fig 4) were distended by deposits of PAS-positive, alcian-blue-positive and mucicarmine-positive material containing non-staining droplets of material which were identified as fat droplets when examined in frozen sections stained with oil red $O$ (Fig 3B). Extensive search failed to show any other type of vascular occlusion and failed to reveal a single example of tumor embolus, although a few microscopic deposits of metastatic tumor were found in areas of brain free of evidence of infarction. 


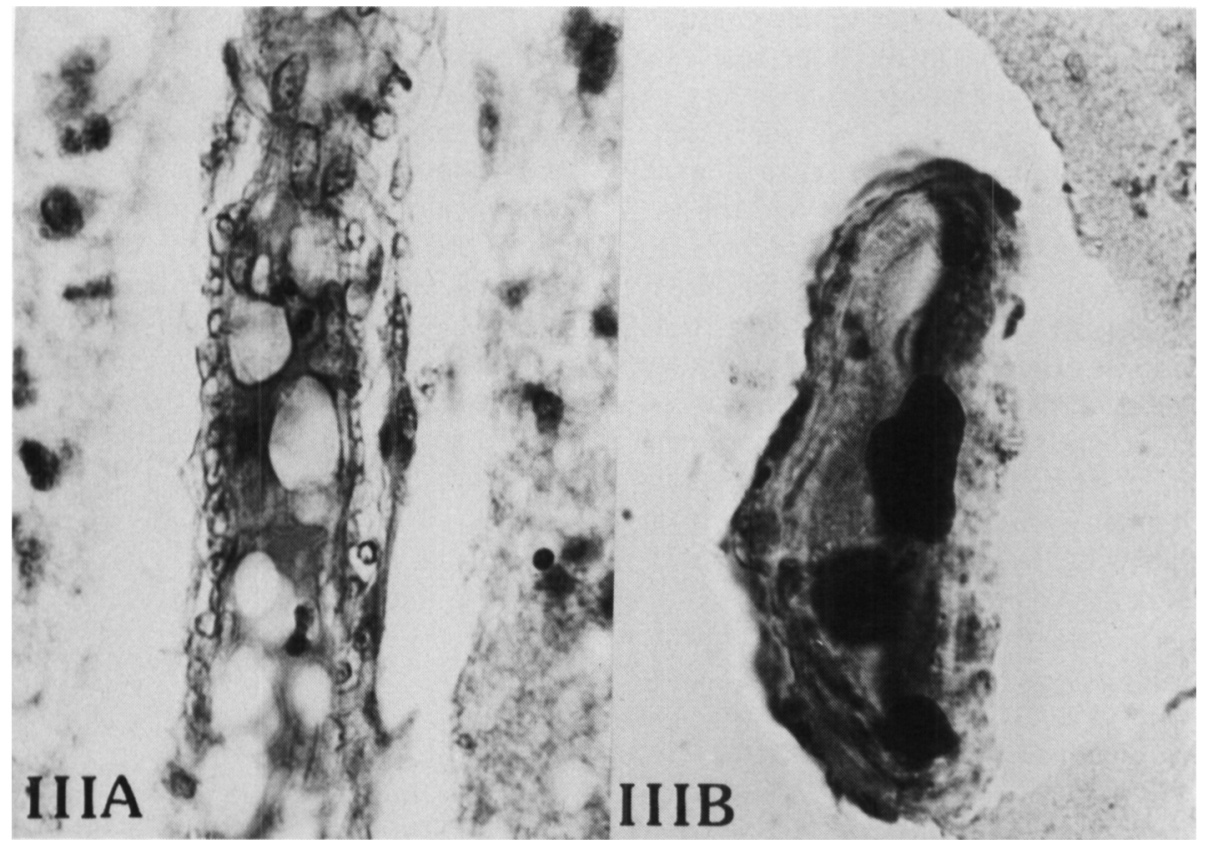

Figure $3 A-$ Small artery in brain showing positive staining of mucin and negative staining of droplet-like spaces. Alcian Blue $x 1000$

$B$-Similar artery to that in $3 \mathrm{~A}$ showing positive staining of fat droplets surrounded by negative staining matrix. The appearance is complimentary to that seen in $3 \mathrm{~A}$. Oil red $0 \mathrm{x}$ 1000

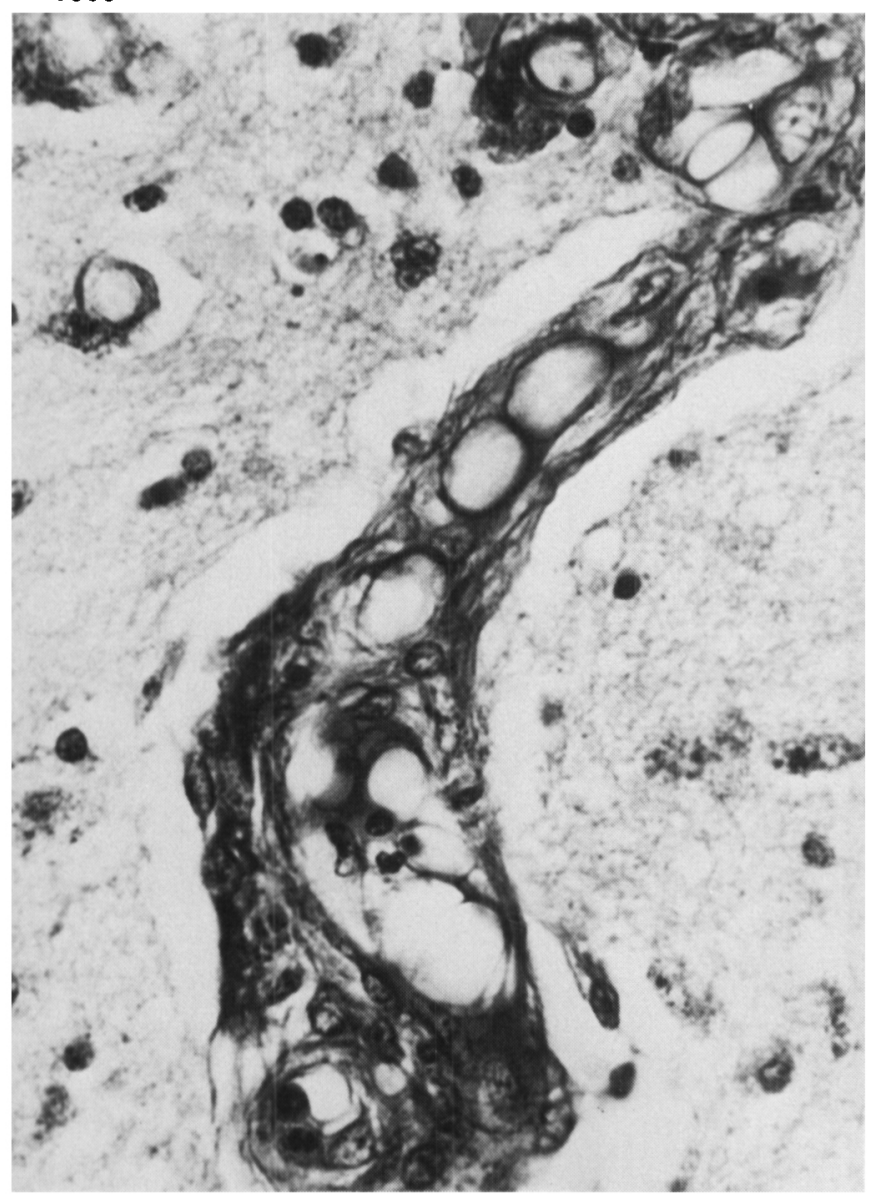

Figure 4-A small artery in brain showing positive staining of mucin. PAS x 1000 the spleen and accumulation of embolized material within the splenic pulp may have been responsible for unusual tissue changes, consisting of small aggregates of macrophages containing mucin and surrounded by a collagenous capsule. The resulting honeycomb pattern completely replaced that of normal splenic pulp (Fig 5). No tumor emboli were identified in any of the above sites.

The extensive tumor deposits in bone showed widespread necrosis possibly as a result of radio and chemotherapy. As a result, many marrow spaces were filled, not with tumor but with lakes of mucin, containing droplets of fat together with a variable amount of connective tissue. Residual normal appearing bone marrow and adipose tissue was also identified. Extensive new bone formation in relation to the tumor deposits corresponded to the radiographic evidence of osteosclerosis.

\section{DISCUSSION:}

That lobular carcinoma of the breast

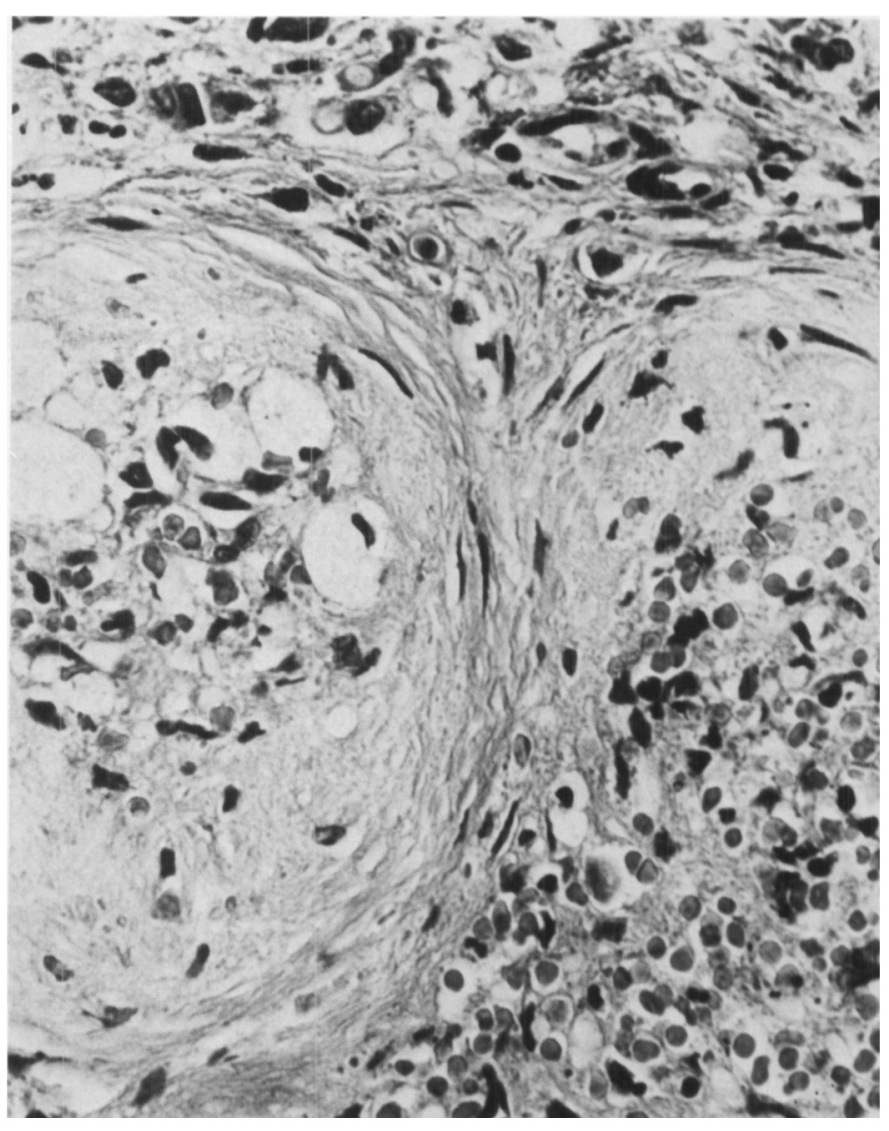

Figure 5-Spleen, showing small nodular areas of sclerosis containing foamy macrophages filled with PAS and Alcian blue positive material. Residual red pulp is seen at lower left, and metastatic mucin containing tumor at right. H\&E x 400 
may be mucin producing has been documented in a recent report (Breslow and Brancaccio, 1976). According to this report, mucin production by such tumors is a quite common occurrence. The pattern of metastasis in the case here reported is not unusual, bone being a particularly common site of metastasis by breast carcinoma. That such metastasis should result in death from embolization is, however, most unusual if not unique. Since mucin embolism seems analogous to fat embolism a brief consideration of fat embolism follows.

The subject of the pathogenesis of fat embolism is controversial, with disagreement among investigators as to the source of the fat (Tedeschi et al, 1968) and the mechanisms whereby fat enters the blood stream (Lehman and Moore, 1927). The classical view (Peltier, 1967), disputed but not disproved (Tedeschi, 1971), is that the fat is derived from adipose cells and occurs when fat is released into extracellular sites by trauma. That embolism occurs chiefly from bone marrow rather than soft tissue adipose deposits is suggested by the commonly accepted association of fat embolism with fractures and by the common histological finding of recognizable marrow in the lung vasculature in such cases.

It has been maintained that bone marrow fat is more likely to embolize than fat from soft tissue sites because the non-collapsing trabecular architecture of the bone promotes entry of fat droplets into veins. Thus, the process of fat embolism can be viewed as comprising two stages, the first, that of release of fat droplets from cells, the second, that of entry of the droplets into the blood stream.

We propose a similar mechanism for the pathogenesis of mucin embolization in this case. The major tumor deposits in this case were in the bone marrow. Tumor necrosis, possibly secondary to anti-tumor chemotherapy, was extensive at this site, with the release of lakes of mucin from tumor cells into the extraceilular space. We cannot be sure how such material enters the blood stream, although we suspect that necrosis of blood vessel walls may have occurred along with the necrosis of tumor cells, permitting entry of the mucin and fat droplets into venous channels which were prevented from collapsing by the trabecular architecture of the bone.

The pattern of effects of the embolization of mucin in this case deserves some attention. The lung appeared to be unaffected. This may be attributed to the minor degree of blockade noted and the protection from ischemic injury afforded by a dual circulation and direct aeration of lung tissue. The kidney showed no evidence of structural injury and the presence of mucin and fat in the urine which might have been expected went undocumented. The spleen was the site of a marked desmoplastic reaction, but the presence of tumor deposits within the spleen casts doubt on embolism as the sole mechanism of tissue injury. Only in the brain were the effects of embolism destructive and clinically important. Since there is little reason to suspect preferential embolism to the brain tissue, damage is attributable to the greater susceptibility of the brain to ischemic injury.

If mucin embolism has occurred as described in this report, what is its prevalance and why has it not been previously recorded? To the question of prevalence, no answer can be given. The requisite combination of a mucin producing tumor, metastasis to bone marrow, and necrosis of such metastases is probably quite uncommon. However, it is also possible that mucin embolism has gone unnoticed since blood vessels seen at post mortem may appear normal unless appropriate stains are done to identify the noncellular content of their lumina. It seems highly unlikely that the case reported here is unique, and it is reported as a mechanism to be considered in obscure cases of tissue infarction.

\section{REFERENCES:}

BRAIN, W. R. and ADAMS, R. D. (1965). In "The Remote Effects of Cancer on the Nervous System (Eds Brain, W, R. and Norris, F. H.) Grune and Stratton, New York.

BRESLOW, A. and BRANCACCIO, M. E. (1976). Intracellular Mucin Production by Lobular Breast Carcinoma Cells. Archives of Pathology and Laboratory Medicine, 100: 620-621.

LEHMAN, E. P. and MOORE, R. M. (1927). Fat Embolism: Including Experimental Production without Trauma. Arch. Surg. 14: 621-662.

PELTIER, L. S. (1967). An appraisal of the problem of fat embolism. International Abstracts of Surgery. 104: 313-318.

TEDESCHI, C. G. and CASTELLI, W., KROPP G. and TEDESCHI, L. G.: (1968) Fat macroglobulinemia and fat embolism. Surgery Gynecology and Obstetrics. 126: 83-90.

TEDESCHI, L. G. CASTELLI, W. P. and TEDESCHI, C. G. (1971). Fat particles in plasma. The macroglobule: its relevance to the concept of fat embolism. Human Pathology 2: 165-171.

WILKINSON, M., CROFT, P. B. and URICH, H. (1976). The remote effects of cancer on the nervous system. Proceedings of the Royal Society of Medicine. 60: 683686. 